identity* $x^{\prime} \alpha f_{\alpha \beta \gamma}=0$ that $[\gamma \alpha, \delta]_{x^{\prime} \beta}-[\beta \alpha, \delta]_{x^{\prime} \gamma}=0$ and hence the difference in question vanishes.

A moment's consideration of the $m$ times extended point transformation will enable us to extend the process to tensors whose components involve derivatives of any order. Thus, if the components $T_{\beta}^{\alpha \ldots}$ of a given tensor are functions of $x, x^{\prime}, x^{\prime \prime}, \cdots, x^{(m)}$, then the quantities

$$
T_{\beta \cdots x(m-1) \gamma}^{\alpha \cdots}-m \cdot T_{\beta \cdots x}^{\alpha \cdots(m) \lambda} \cdot\left\{\begin{array}{c}
\lambda \\
\dot{\gamma}
\end{array}\right\}
$$

are the components of a tensor. The commutative property established above holds in this case also.

The University of TeXas

\title{
ON THE DIVISIBILITY OF LOCALLY CONNECTED SPACES $\dagger$
}

BY G. T. WHYBURN

1. Introduction. In this paper a property of connected, locally connected, separable metric spaces will be established which is stronger than that of divisibility in the sense of W. A. Wilson. In order to distinguish our property from that of Wilson we shall use the term strong divisibility.

A space $M$ will be said to be strongly divisible if for every pair of mutually exclusive closed and connected subsets $A$ and $B$ of $M$ there exists a decomposition of $M$ into three mutually exclusive sets $R, F$, and $G$, where $R$ and $G$ are connected and open and contain $A$ and $B$, respectively, and where $F$ is the common boundary of $R$ and $G$, that is, $F=F(R)=F(G)$.

2. Theorem. Every connected, locally connected, separable, metric space $M$ is strongly divisible.

* J. H. Taylor, loc. cit., equation (14), p. 253.

$\dagger$ Presented to the Society, December 31, 1930.

$\ddagger$ According to Wilson, a space $M$ is divisible if for every pair of mutually exclusive subcontinua $A$ and $B$ of $M$ there exists a decomposition of $M$ into two continua $P$ and $Q$ such that $P \cdot B=Q \cdot A=0$. See this Bulletin, vol. 36 (1930), p. 85. Wilson's theorem that every connected, locally connected, separable metric space is divisible is obviously an immediate corollary to our theorem below in $\$ 2$. 
Proof.* Let $A$ and $B$ be any two mutually exclusive closed and connected subsets of $M$. We may suppose the metric so defined in $M$ that $\rho(A, B)=d>0$, or in other words, $M$ is homeomorphic with a space $M^{\prime}$ in which this is true. $\dagger$ For each positive number $r<d$, let $S_{r}(A)$ and $V_{r}(A)$, respectively, denote the set of all points whose distances from $A$ are equal to and less than $r$. Let $U_{r}(A)$ denote the component of $V_{r}(A)$ containing $A$, let $G_{r}(B)$ be the component of $M-\overline{U_{r}(A)}$ containing $B$, let $X_{r}=F\left[G_{r}(B)\right]$, and let $R_{r}(A)$ be the component of $M-X_{r}$ containing $A$. Then clearly we have

$$
X_{r}=F\left[G_{r}(B)\right]=F\left[R_{r}(A)\right] \subset F\left[U_{r}(A)\right] \subset S_{r}(A) .
$$

Now if $r_{1}<r_{2}<d$, we have

$$
G_{r_{2}}(B)+X_{r_{2}}=\overline{G_{r_{2}}(B)} \subset G_{r_{1}}(B),
$$

and therefore

$$
\overline{R_{r_{1}}(A)} \subset R_{r_{2}}(A) \text {, for } R_{r_{1}}(A) \cdot X_{r_{2}}=0 \text { by (ii). }
$$

Now let $E$ be the set of all positive numbers $e<d$ such that there exists at least one component $D_{e}$ of $M-X_{e}$ which is distinct from $R_{e}(A)$ and from $G_{e}(B)$. Then if $e_{1}$ and $e_{2}$ are numbers. of $E$ and $e_{1}<e_{2}$, it follows by (2) and (3) that $D_{e_{2}} \subset G_{e_{1}}(B)$, because $F\left(D_{e_{2}}\right) \subset X_{e_{2}}$. Hence $D_{e_{1}} \cdot D_{e_{2}}=0$. Therefore $E$ must be countable, for the sets $D_{e}$ are open. It follows that there exists a positive number $f<d$ which does not belong to $E$ and hence such that

$$
R_{f}(A)+G_{f}(B)+X_{f}=M,
$$

and thus we have a strong division of $M$ between $A$ and $B$.

CoROLlaRY. If the space $M$ as in our theorem is locally compact, then each non-cut point $p$ of $M$ is contained in an arbitrarily

* Considerable similarity will be noted between this proof and a number of proofs for closely related theorems recently published by the author. See, for example, my paper Non-separated cuttings of connected point sets, Transactions of this Society, vol. 33 (1931).

$\dagger$ In view of the theorem of Tychonoff, Mathematische Annalen, vol. 95, pp. 139-142, that our space $M$ is normal, a glance at Urysohn's proof for the existence of a metric in normal, perfectly separable, Hausdorff spaces is sufficient to verify this statement. See P. Urysohn, Zum Metrisationsproblem, Mathematische Annalen, vol. 94, pp. 309-315 . 
small connected neighborhood $R$ whose exterior $G$ is connected and whose boundary $F$ is the boundary also of $G$.

For there exists* an arbitrarily small neighborhood of $p$ whose complement $B$ is connected. Then setting $p=A$ and applying our theorem we have the desired result.

3. Conclusion. In conclusion we mention some consequences of our theorem and corollary.

In the first place, it is to be noted that, by virtue of our corollary, we can define the term region in any non-compact but locally compact separable metric space $S$ which is connected and locally connected and has no cut point in such a way that the topological character of the space is not altered and so that axioms 1-5 and $7^{\prime}$ of R. L. Moore's $\dagger$ system $\sum_{2}$ are satisfied. For this purpose it is only necessary to say that a region is a compact connected open set of points $R$ whose exterior $S-\bar{R}$ is connected and has the same boundary as $R$. Thus it is seen that these axioms of Moore's set $\sum_{2}$ restrict the space only to the extent of making it a non-compact cyclicly connected continuous curve.

It is obvious that each point (whether a cut point or not) of a space $M$ as in our corollary is contained in an arbitrarily small neighborhood $R$ whose exterior is the sum of a finite number of connected sets and has the same boundary as $R$.

We note also the following application. Let $J$ be any simple closed curve contained in the space $M$ of our theorem, let $a$ and $b$ be any two points of $J$ such that $M-(a+b)$ is connected, and let $t_{1}$ and $t_{2}$ be the two arcs of $J$ from $a$ to $b$. Then from our theorem it follows that there exists a decomposition of $M$ into three sets $R_{1}, R_{2}$, and $F$, where $R_{1}$ and $R_{2}$ are connected and open and contain $t_{1}-(a+b)$ and $t_{2}-(a+b)$, respectively, and $F$ is the common boundary of $R_{1}$ and $R_{2}$ and hence contains $a+b$. To prove this it is only necessary to note that in the space $M-(a+b)$, the sets $t_{1}-(a+b)$ and $t_{2}-(a+b)$ are closed and of course connected and mutually exclusive.

The Johns Hopkins University

* See H. M. Gehman, Proceedings of the National Academy of Sciences, vol. 14 (1928), pp. 431-432, and W. L. Ayres, Monatshefte für Mathematik und Physik, vol. 36 (1929), pp. 139-140.

$\dagger$ See Transactions of this Society, vol. 17 (1916), p. 163. 